\title{
EL ROL DE LA FAMILIA EN LA CALIDAD DE VIDA Y LA AUTODETERMINACIÓN DE LAS PERSONAS CON TRASTORNO DEL ESPECTRO DEL AUTISMO *
}

\section{THE ROLE OF THE FAMILIY IN THE QUALITY OF LIFE AND SELF-DETERMINATION OF PEOPLE WITH AUTISTIC SPECTRUM DISORDER}

\author{
Manoel Baña Castro \\ Departamento de Psicología Evolutiva y de la Educación, Universidad de A Coruña. España
}

Palabras Clave: trastorno del espectro del autismo; trastorno generalizado del desarrollo; calidad de vida; autodeterminación

Key Words: Autistic Spectrum Disorder; Pervasive Developmental Disorder; Quality of Life; Self-determination

*Nota de la Editora: Esta Comunicación es la que ha dado lugar a Conferencias y Ponencias del Prof. Baña en nuestro país,
quien generosamente ha tomado la tarea de enviarnos sus notas inéditas sobre las que ha construido sus disertaciones.

\section{Las personas con Trastorno del Espectro del Autismo y sus familias}

Los Trastornos del Espectro del Autismo (TEA) comprenden una serie de alteraciones graves que afectan a la interacción social, el comportamiento y la comunicación desde los primeros años de la infancia. Son de carácter generalizado y afectan al desarrollo en su conjunto, acompañando a la persona durante todo su ciclo vital (Frith, 2003). "La etiqueta de TEA parece remitir a un conjunto enormemente heterogéneo de individualidades, cuyos niveles evolutivos, necesidades educativas y terapéuticas, y perspectivas vitales son enormemente diferentes..." (Riviere, 2000).

Sin embargo, muchas veces cuando nos referimos a estos trastornos que inciden en el desarrollo, confundimos a éstos con las personas que los poseen o viven con ellos. Es decir, todas las personas con TEA son diferentes, no están caracterizadas por los rasgos de estos trastornos, sino que se caracterizan por el entorno en el que viven, las experiencias que tienen en sus vidas $y$, sobre todo, por los apoyos que tengan para superar las dificultades de estas experiencias y los modelos educativos a los que estén sujetos. De esta dificultad, por generar un marco teórico común, Frith (1991) señala el concepto de "Espectro Autista" para recoger alteraciones, dificultades y trastornos con la sintomatología del trastorno autista. El DSM5 recoge estas consideraciones en su Manual de Diagnóstico e incorpora el de TEA como general para referirse a una serie de trastornos con una sintomatología común como la dificultad de comunicación o su comportamiento poco adaptativo en general. Nos referirnos a un grupo de personas que viven y forman parte del sistema social, comunitario, familiar, escolar. Si nos referimos a las familias y el entorno familiar se estará señalando las funciones que el grupo familiar tiene para con las personas que conviven en él: cuidar y velar por su supervivencia, educarlas y formarlas para vivir en la comunidad social de referencia o en otras más o menos allegadas; el papel educador familiar no tuvo importancia hasta hace poco en las personas con Trastorno del Espectro del Autismo, sobre todo, debido al modelo clínico-médico que prepondera incluso en estos momentos; el modelo sistémico/social aporta gran importancia a la familia y a su papel educativo-estimulador, también normalizador-inclusor; es el grupo de referencia que permite los primeros aprendizajes sociales y permite incluirse, poco a poco, en un clima adecuado y lúdico en la sociedad en que

Correspondencia: Manoel Baña Castro. Director de la Unidad Clínico-Asistencial de Trastornos del Espectro del Autismo y Trastornos del Desarrollo. Correo Electrónico: mbcastro@udc.gal 
se inserta; este papel familiar es imprescindible donde los valores y actitudes y normas sociales no tienen un claro exponente y tienden a ser difusas o confundirse. El cambio social da lugar a un cambio familiar por lo que la familia juega un papel de referencia al ser el grupo primario más representativo y de referencia social. En el caso de la Trastorno del Espectro del Autismo pasa a ser imprescindible su aportación educativa puesto que facilita los apoyos y oportunidades para el desarrollo de las personas más allá de sus alteraciones y dificultades.

La familia pasa a ser el principal y más permanente apoyo para el individuo, de su actuación van a depender muchas de las expectativas, posibilidades y bienestar de la persona. Desde hace algún tiempo se destaca el trabajo con las familias con la intención de reconocer la importancia del papel educativo y socializador de los padres, madres, hermanas, abuelos... Conocer el contexto familiar es de suma importancia para analizar el desarrollo de las personas con Trastorno del Espectro del Autismo y estimular su aprendizaje potencial. El nacimiento de una persona con Trastorno del Espectro del Autismo en la unidad familiar cambia las expectativas e influye en el grupo familiar, afectando al desarrollo de la persona con el desorden y la relación entre las redes de apoyo que se puedan poner en marcha y la pretendida calidad de vida.

La aparición de una persona con Trastorno del Espectro del Autismo en la familia supone una alteración; si bien a lo largo de muchos años predominó una visión deficitaria, patológica y excepcional sobre estas personas, en las que la educación quedaba en las manos de especialistas centrándose en la persona y relegando a un segundo plano el papel de la familia o dedicándolo a cuidados y atención meramente instrumental, es decir, se buscaba cómo podría contribuir la familia e incorporarse a programas específicos profesionales; en este caso, la familia era una prolongación del trabajo de profesionales, pudiéndose especializar en tratar y/o estimular a las personas con Trastorno del Espectro del Autismo, suponiendo una carga y/o dedicación exclusiva más. Progresivamente, la evolución hacia una visión más global e interactiva, basada en las necesidades y demandas de la personas y del grupo familiar, abarca la gran diversidad de las familias así como la del Trastorno del Espectro del Autismo de las personas (Paniagua, 1999). Por lo tanto se pasa de una concepción de corte patológico, centrada en el déficit a otra educativa en la que el eje son las necesidades y demandas familiares.

La concepción de las necesidades educativas en una visión global sistémica o interactiva concibe a las familias de personas con Trastorno del Espectro del Autismo, como familias típicas en circunstancias excepcionales. Las necesidades no sólo están determinadas por la discapacidad del hijo/a y otras variables familiares sino también en gran medida, por la respuesta o falta de respuesta en un medio social determinado (Paniagua, 1999). Dentro de este ámbito valorar como es la evolución de las familias que tienen una persona con Trastorno del Espectro del Autismo pasa a ser relevante. Algunos autores llegan a equiparar las reacciones emocionales que se pueden encontrar en las familias hasta la aceptación de una persona con Trastorno del Espectro del Autismo, cómo similares a las de un duelo por la muerte de un ser querido. Supuestamente hacen mención a la persona que esperaban y no apareció y la que encontraron y no esperaban con respeto a las posibles expectativas sociales, comunitarias e individuales con respecto a un nuevo miembro del grupo. Estos autores hacen referencia a un modelo de adaptación desde que las familias tienen constancia del Trastorno del Espectro del Autismo hasta que llegan a la aceptación. A este respecto, Cunningham y Davis (1988) señalan las siguientes fases:

- Fase de Shock. Se caracteriza por la conmoción o bloqueo

- $\quad$ Fase de no. Los padres ignoran el problema o actúan en el día a día como si nada hubiese ocurrido.

- Fase de reacción. Los padres intentan comprender la discapacidad y se basan en las interpretaciones que ellos mismos hacen de la misma.

- Fase de adaptación y orientación. Es una fase más realista y práctica, centrada en lo que se debe hacer en torno a las necesidades del hijo y en la mejor ayuda que se le pode ofrecer.

En relación a este tema, Giné (2001) señala que el nacimiento de un hijo con Trastorno del Espectro del Autismo provoca siempre, en mayor o menor medida, una crisis que se caracteriza por: a) un fuerte impacto psicológico y emocional; b) un proceso de adaptación y redefinición del funcionamiento familiar; c) cambios en la relación de pareja; y d) la necesidad de ayuda y de asesoramiento. La interacción de los padres con el hijo 
depende del tipo y grado de retraso, de la rapidez en la toma de conciencia, de la naturaleza de los desafíos que le plantea la atención al hijo/a y de la historia, recursos y características de la familia en su conjunto (Aierbe, 2005).

Ahora bien, no se puede suponer que todas las familias atraviesen una secuencia similar de etapas; en realidad, las familias reaccionan de maneras muy diversas ante la aparición de una persona con Trastorno del Espectro del Autismo (Heward, 2000). Para conocer mejor las actitudes y comportamientos familiares resulta útil basarse en el concepto de ciclo vital familiar. Freixa (1993) entiende por ciclo vital la progresión de estadios del desarrollo mas o menos estables, separados por etapas de transición, períodos relativamente breves de desorganización, marcados por ceremonias, rituales y acontecimientos que señalan la entrada a un nuevo estadio del desarrollo.

Asimismo, Gallimore, Coots, Weisner, Garnier y Guthrier (1996) señalan el proceso de acomodación de la familia al desarrollo del niño/a cómo similar al resto de las familias; si bien los padres y madres tienen que ser más selectivos a la hora de plantear las actividades para el cuidado de sus hijos/as con Trastorno del Espectro del Autismo y además las rutinas son más complejas. En general, los estudios advierten de la necesidad de considerar los efectos de las ideas, intenciones o metas de los padres y educadores, como uno de los elementos determinantes de las pautas de interacción a desarrollar. Estos autores afirman que la acomodación que realiza la familia ante las dificultades del niño/a presentan un programa mixto de continuidad y de cambio, con momentos de transición que pueden generar mayor estrés. Los factores que intervienen en la variedad de respuestas adaptativas de las familias son: Las características del niño/a, las creencias de la familia sobre el desorden de la persona están muy relacionadas con el estrés parental, el ajuste familiar, la angustia psicológica, la calidad de las relaciones familiares y los sistemas de apoyo y recursos externos.

Por otra parte, Guralnick $(1997 ; 1998)$ señala que la presencia de niños/as nacidos con riesgo biológico o condiciones manifiestas de Trastorno del Espectro del Autismo, genera situaciones potencialmente estresantes adicionales con las que la familia se tendrá que enfrentar. Señala cuatro aspectos:

1. Obtener información relativa al desarrollar y salud de su hijo/a.

2. Hacer frente a la angustia que se genera a partir del riesgo biológico o de Trastorno del Espectro del Autismo.

3. Realizar mayor demanda de servicios y recursos que de otra manera no sería precisos, lo cual puede alterar y estresar las rutinas de la familia.

4. Afrontar la disminución de la confianza de la familia en sus posibilidades educativas y también de su autoestima.

- Para disminuir el estrés familiar, la intervención iría dirigida a:

- Aumentar la sensibilidad de las familias cara las posibilidades y competencias de sus hijos/as.

- Estimular la participación del hijo con desorden en rutinas diarias de la familia.

- Promover en la familia el desarrollo de actitudes y valores sensibles a la diferencia.

- No anteponer el saber experto del profesional al saber educativo de las propias familias.

- Incidir en factores protectores de los impactos negativos de la crianza de estos niños: mejores relaciones familiares, crear estilos de afrontamiento al estrés adecuados, ampliar redes de apoyo a los padres...

Las familias que tienen miembros con Trastorno del Espectro del Autismo deben desempeñar mayor número de tareas y papeles que otras familias que no tienen estas características. En la investigación sobre este tipo de familias, muchas veces destaca el papel de la tensión que padece la familia con miembros con Trastorno del Espectro del Autismo. Pero la adaptación o la falta de ajuste familiar no depende exclusivamente de la presencia o ausencia de la tensión sí no que es la interacción entre el suceso estresante, los recursos de la familia y la estimulación de la seriedad del suceso, lo que determina el grado en que la familia será vulnerable a la tensión y la crisis.

La mayor parte de las familias en las que viven niños o adultos con Trastorno del Espectro del Autismo no presentan problemas graves de adaptación a la tensión. Por tanto, podría hablarse de la existencia de una serie de aspectos de la persona y el Trastorno del Espectro del Autismo (tipo y gravedad del Trastorno del Espectro del Autismo, extensión, duración...) de la situación y contexto en el que se da (interacción padreshijos/as, apoyo social, recursos familiares...) que pueden influir para agravar o aliviar la potencial tensión en la que la familia se puede encontrar inmersa.

Que no sean frecuentes las situaciones de tensiones no implica que las familias con 
hijos/as con Trastorno del Espectro del Autismo no tengan necesidades de apoyo. Por el contrario, el hijo/a con Trastorno del Espectro del Autismo implica muchas más necesidades y demandas de apoyo personal y social que el hijo/a sin Trastorno del Espectro del Autismo, pues debe superar muchas más situaciones difíciles. Además, a mayor Trastorno del Espectro del Autismo, en extensión, gravedad o duración, las necesidades de apoyo se multiplican. A cuyo objeto tiene sentido preocuparse por hablar sobre la calidad de vida de las familias junto con la calidad de vida de los hijos/as con Trastorno del Espectro del Autismo, desde edades tempranas.

\section{La familia en la calidad de vida e independiente de la persona con Trastorno del Espectro del Autismo}

En las primeras edades, el papel de la familia es fundamental para conseguir el progreso de sus hijos/as, ya que su influencia educativa dejara sentir en la relación y comunicaciones precoces y en posibilitar la participación de los niños/as en las actividades propias de las rutinas diarias. Por otra parte, si se tiene en cuenta que el nacimiento de un niño/a con Trastorno del Espectro del Autismo provoca en la familia un fuerte contratiempo emocional que puede perjudicar la relación con el hijo/a, se deberá contemplar la necesidad de procurar ayuda y orientación a la familia desde el primer momento (Aierbe, 2005; Giné, 2001). Para disponer de esta ayuda es preciso señalar que a lo largo de la infancia y adolescencia es necesario determinar las experiencias y oportunidades que se le proporcionan a los chicos mediante el aprendizaje escolar. Se pueden remarcar cómo aspectos importantes en esta etapa: la inclusión escolar que debe implicar a todos los alumnos/as en el entorno educativo y la transición a la vida adulta, incluyendo para ello la formación profesional y objetivos de tipo personal y social.

Según West (1986), en las familias con hijos/as con Trastorno del Espectro del Autismo las etapas del ciclo vital familiar se prolongan. Como el niño/a con Trastorno del Espectro del Autismo crecerá y se desarrollará más atípicamente, precisará que la familia esté pendiente de tareas normalmente asociadas al cuidado de un chico/a de menor edad.

Para Paniagua (1999), estas implicaciones que tiene para la familia con un niño/a con Trastorno del Espectro del Autismo son: a) una preocupación por el presente y el futuro del hijo/a; b) decidir tratamientos médicos, elección de profesionales y opciones educativas; c) el aumento de dedicación: cuidados físicos, tiempo de interacción, más situaciones de juego y estudio compartido, programas de estimulación temprana, actividades de ocio y refuerzo de la escolarización; e) gastos extraordinarios (de tipo médico, ortopédico, educativo, rehabilitador); y f) futuro lleno de interrogantes e incertidumbre ante la venida independiente del hijo/a con Trastorno del Espectro del Autismo. No obstante, muchas familias consiguen grandes cuotas de satisfacción y adaptación, siendo la conclusión más ampliamente aceptada que el impacto es menor si ambos padres participan igualitariamente en la ejecución tanto de las labores del hogar como del cuidado de los hijos/as con Trastorno del Espectro del Autismo.

Con la llegada de la vida adulta es necesario plantearse una serie de necesidades como: el trabajo (la inclusión laboral en puestos de trabajo ordinario, el empleo con apoyo, la adaptación del puesto de trabajo), la vida afectiva (la educación sexual, la higiene, la prevención, los valores...), el hogar (las viviendas tuteladas...), la participación social (emplear los servicios comunitarios, culturales, deportivos, religiosos, de ocio...)

No existe un patrón de comportamiento generalizable a todos los niños/as con Trastorno del Espectro del Autismo y tampoco existe un patrón aplicable a todas las familias. Por ello, se puede decir que la gran variedad de familias se puede observar en el impacto de la presencia de un hijo/a con Trastorno del Espectro del Autismo en el comportamiento de la familia para responder a sus necesidades.

El ámbito de las actitudes y comportamientos de las familias de personas con Trastorno del Espectro del Autismo generó muchas publicaciones; en opinión de Molina (1999) la mayoría no están basadas en investigaciones rigurosas y las que se basan en datos empíricos ofrecen datos bastante contradictorios. A este respeto, Lambert y Rondal (1982), tras realizar encuestas a padres de personas con discapacidad, señalan que los cinco factores más importantes que condicionan las actitudes y el comportamiento de las familias son: - El modo en el que los padres se enteran del diagnóstico y la calidad de las informaciones y el apoyo psicológico que han recibido desde el nacimiento del niño/a. 
- La edad del niño/a en el momento en que fueron conscientes de la alteración del desarrollo del hijo/a.

- El nivel socioeconómico y cultural de los padres.

- La situación de la constelación familiar antes de nacer el niño/a con Trastorno del Espectro del Autismo, sobre todo en lo que respeta al clima afectivo entre la pareja.

- Los recursos de asesoramiento y de apoyo que la sociedad está en condiciones de ofrecer a la educación de estos chicos y chicas. Tomkierwicz (1979) divide estos factores en tres grupos: subjetivos, objetivos y sociales. Los factores subjetivos incluyen los mecanismos de defensa (repulsa, culpabilidad, frustración, carencia de expectativas, indiferencia, negatividad, heteroagresividad, autoagresividad). Entre los factores objetivos, los más destacados son: la etiología de la alteración, la morfología y los trastornos asociados. Finalmente, dentro de los factores sociales señala: el nivel socioeconómico, los prejuicios sociales y las actitudes de la familia. En lo relativo a las relaciones fraternales, es preciso destacar que es uno de los ámbitos menos estudiados dentro de las relaciones intrafamiliares. Existen lagunas muy importantes en este ámbito derivadas de la no utilización del enfoque sistémico en las investigaciones sobre las relaciones fraternas entre chicos/as y sus hermanos con alteraciones (Freixa, 1993). En general, se acepta la idea de que el impacto de un miembro con Trastorno del Espectro del Autismo sobre sus hermanos depende del clima social familiar y de las relaciones entre padres-hijo con Trastorno del Espectro del Autismo (Molina, 1999).

La necesidad de dar apoyo y orientar a las familias con hijos/as con Trastorno del Espectro del Autismo, se sustentan en dos razones: 1) Ayudar a los padres en el proceso de adaptación que deben efectuar para que el clima emocional y de relación con el hijo/a no se resienta lo más mínimo, y 2) Facilitar a los padres que puedan hacerse cargo del papel que tienen en la educación del hijo con Trastorno del Espectro del Autismo, sobre todo durante los primeros años. Según Aierbe (2005) tanto la experiencia como la investigación coinciden en señalar la importancia de priorizar el trabajo con las familias como una vía privilegiada para contribuir positivamente al desarrollo de los niños/as con Trastorno del Espectro del Autismo.

La intervención psicoeducativa en las familias de personas con Trastorno del Espectro del Autismo resulta de gran importancia debido a que se está produciendo una mayor concienciación a la hora de apoyar a labor educativa familiar. Dentro de este ámbito se le presta especial atención a la intervención temprana ya que se defiende la idea de que cuanto menor es la edad del niño, más eficaz es la intervención en su familia porque es en las primeras edades cuando se produce un avance importante en el proceso de socialización e individualización y se producen las bases básicas para ello. Asimismo, muchos de los problemas que se detectan en el ámbito de la infancia requieren de un tratamiento preventivo en el que los distintos servicios coordinen esfuerzos y atiendan simultáneamente a las necesidades de los niños/as y sus familias.

En la actualidad se está produciendo un cambio de paradigma en la concepción de la atención precoz. Pasará de un modelo casi exclusivamente centrado en el niño/a, con una finalidad más rehabilitadora a un modelo centrado en la familia como contexto del desarrollo y orientado a optimizar la interacción a partir de las rutinas diarias. Los patrones de interacción que se manifiestan en las actividades propias de las rutinas diarias influyen poderosamente en la dirección que tomará el desarrollo del niño/a (Gallimore, Weisner, Kaufman, \& Bernheimer 1989; Gallimore, Weisner, Bernheimer, Guthrie, \& Nihira, 1993). Según Giné (2001) la necesidad de procurar desde los primeros momentos ayuda y orientación a la familia con algún hijo/a con Trastorno del Espectro del Autismo está plenamente justificada, dada la importancia, por un lado, de la relación y comunicación más precoces y la participación del niño en las actividades propias de las rutinas diarias, por otro, que la aparición de una persona con Trastorno del Espectro del Autismo con alteraciones en su desarrollo provoca en la familia un impacto emocional que puede afectar la relación con su hijo/a.

Desde el modelo comprensivo de intervención, Guralnick $(1997 ; 1998)$ indica los siguientes factores básicos a la hora de regir el curso del desarrollo de un niño/a:

- Factores próximos. Son aquellos que condicionan el progreso del niño/a de forma conjunta o separada. Incluyen tres patrones de interacción familiar.

- La calidad de las transacciones padres/ hijo/a.

- Las experiencias con el entorno físico y social que los miembros de la familia ofrecen al niño/a. 
- Los hábitos de la familia que aseguran la salud y seguridad del niño/a; atención sanitaria; alimentación y ausencia de violencia.

- Factores distales están constituidos por las características de la familia y distinguen:

- Las características personales de los padres: nivel de educación, la salud, el equilibrio emocional, las creencias y valores culturales y la manera de afrontar los problemas

- Las características de la familia, no directamente relacionadas con la alteración o situación de riesgo del niño/a, como: la calidad de la relación de pareja, los recursos disponibles y la facilidad de acceso a redes de apoyo. Desde una perspectiva profesional y de las organizaciones conviene situar los objetivos del trabajo en el ámbito familiar de las personas con Trastorno del Espectro del Autismo dado que se trata de un ámbito mucho más amplio y eficaz que una visión estrictamente individual, psicopatológica y de intervención terapéutica. Este planteamiento debe hacerse tanto en la evaluación de la situación y necesidades de la familia como para establecer objetivos de intervención y prioridades de la misma. La perspectiva es generar estructuras de pensamiento y trabajo en un plano de colaboración familias-profesionales, en lugar de establecer jerarquías profesional-familia en la que esta última es solamente receptora pasiva de las orientaciones e intervenciones. La participación activa de la familia en todas las fases del proceso de atención de la persona con Trastorno del Espectro del Autismo es fundamental, lo que no debe obviar el estudio de las necesidades de apoyo de la propia familia.

En este sentido, el Trastorno del Espectro del Autismo puede entenderse desde el impacto que causa en la vida familiar o desde el efecto del Trastorno del Espectro del Autismo en el individuo y en el papel a desempeñar por la familia. En el primer caso, corresponde examinar las transformaciones que ocurren en el seno familiar y generar servicios de apoyo a la familia en las diferentes fases de evolución del individuo con Trastorno del Espectro del Autismo, siendo el objeto de atención de la propia familia. En el segundo caso, el usuario de los servicios es la persona con TEA y el papel de la familia es la colaboración estrecha con los profesionales.

A través del estudio de este tipo de familias se puede apreciar que los factores que afectan a la vida en las personas con Trastorno del Espectro del Autismo presentan muchas interrelaciones con los referidos a la familia. Además, parece que los valores sociales, imágenes y estereotipos se extienden más allá de las personas con Trastorno del Espectro del Autismo hacia sus familias, quienes deben ofertar una gran dosis de fuerza, flexibilidad y creatividad en los procesos de adaptación y colaboración.

Los contenidos en torno a los que se debe articular la tarea con las familias desde una perspectiva educativa son: educar a los padres; ofrecer ayuda emocional; asesoramiento en planes y objetivos educativos y la participación de los padres en el movimiento asociativo y en la defensa de sus derechos (Giné, 2001). Esta tarea puede realizarse desde redes de apoyo social que faciliten una correcta inclusión individual y familiar.

\section{La educación familiar en las personas con Trastorno del Espectro del Autismo}

Educar a las personas con Trastorno del Espectro del Autismo implica tener en consideración la atención educativa que se les ofrece a largo de su vida familiar, escolar y social-comunitaria. Esta atención educativa es fundamental no solo para el desarrollo de la persona además de ser un elemento enriquecedor donde se reconoce el valor de la diversidad como un bien en sí mismo. En la escuela se aprende a vivir con los demás en situaciones normalizadas y los demás aprenden a aceptar la diferencia como un elemento que facilita el progreso de la sociedad, la riqueza y contribución del diferente. Algunos de los principios relevantes para esta educación para el desarrollo son:

- El de normalización desenvuelto por Wolfensberger (1972) que señala que normalizar es la utilización de medios, culturalmente tan normativos como es posible, en orden a establecer y/o mantener conductas y características personales que son tan culturalmente normativas como posibles. Al igual que no se puede dudar que la escolarización obligatoria es una conquista, la normalización es un logro para las personas con Trastorno del Espectro del Autismo (García Sánchez, 2005). Este principio está sujeto a muchas críticas en el ámbito educativo. En 1990 Mesibov señala que el hecho de adherirse al principio de normalización no significa que se esté practicando. Para ello, Mesibov (1990) propone una vuelta a las necesidades individuales de las personas (...) entiende la normalización no como un objetivo sino como una forma de implementar estrategias que valoren y acepten a las perso- 
nas con trastornos, que no las estigmatice, que promuevan el desarrollo propio y consideren el potencial de los educadores. Estas alternativas suponen la tolerancia de la ambigüedad y variabilidad en una dinámica negociada de clarificación de responsabilidades y roles (García Sánchez, 2005; Kebbon, 1993). Otra de las críticas al principio de normalización procede de los conflictos que se asumen desde la perspectiva del poder o de la ausencia del poder, en relación con la sociedad multirracial y multicultural. El principio de normalización es simplista al asumir niveles homogéneos en las personas capaces y en conductas aceptables y considera devaluadas a las personas con Trastorno del Espectro del Autismo. Es ingenuo asumir que el simple contacto llevará la aceptación de las otras personas. Es también ilusorio no valorar ni poner en entredicho los valores sociales. La esencia de la normalización no reside en un programa determinado de tratamiento, sino en proporcionar a las personas diferentes socialmente la dignidad completa que les corresponde como derecho propio (Perrin \& Nirje, 1985). Esto implica la puesta en marcha de muchas actividades más allá de las tareas concretas de la rehabilitación, física, sensorial o cognitiva de un individuo. La afectación de la vida familiar, profesional y social de la persona con Trastorno del Espectro del Autismo debe ser estudiada y planificar actuaciones que permitan su restauración, recuperación, recreación, reinvención y/o readaptación. La calidad de vida puede considerarse en parte una extensión lógica del concepto de normalización pues tiene implicaciones sobre las directrices de los estándares de servicios y condiciones de vida (Taylor \& Brown, 1988). Como explica Alba (1994) el concepto de normalización debe entenderse no como un intento de conseguir que todas las personas sean etiquetadas cómo típicas si no que, mediante la utilización de determinados recursos, sea posible que cada persona pueda llevar una vida autónoma e independiente como miembro de esta sociedad.

La perspectiva ecológica concibe al principio de normalización como una intervención, un esfuerzo en la transformación del entorno y que este sea el más idóneo y satisfactorio para las personas con Trastorno del Espectro del Autismo al ver que es satisfactorio para la sociedad y todo ello sin renunciar a la busca de alternativas e innovaciones específicas. En este sentido, Bronfrenbrenner señala que el desarrollo humano tiene lugar en el contexto de las relaciones familiares y ese desarrollo es el resultado no sólo de los factores ontogénicos si no también de la interacción de la dotación genética de la persona con el entorno inmediato familiar y, eventualmente, con otros componentes del ambiente. Según Bronfenbrenner (1979) comprende el estudio científico de la progresiva acomodación mutua entre un ser humano activo, en desarrollo y las propiedades cambiantes de los entornos inmediatos en las que vive la persona, en cuanto este proceso se ve afectado por las relaciones que se establecen entre estos entornos y los contextos más grandes en los que están incluidos los entornos.

- El principio de inclusión entendido como el cambio social y por extensión una transformación del sistema educativo, que debe ser capaz de valorar la diversidad de forma que cada persona pueda ser respetada y asumida, con independencia de sus condiciones personales, sociales o culturales. El concepto inclusión se refiere a modificar actitudes negativas y estereotipos, calificar al profesorado y a otros profesionales, cambiar las dinámicas de programación educativa y el diseño curricular habitual, y muchas otras acciones (Verdugo \& Rodríguez, 2008).

El principio de inclusión educativa se vincula a la dignidad de las personas como derechos inalienables y a la gran meta de comprender la acción escolar en la tarea de ayudar a superar las discriminaciones y contribuir a la compensación de las desigualdades de distinto tipo (Echeita et al., 2008).

El derecho a una educación inclusiva implica no ser discriminado por razones de diferencias o alteraciones entre otras, sólo se podrá ir consiguiendo cuándo se reconozcan y se eliminen las barreras escolares de distinto tipo y en distintos planos aún existentes en nuestro sistema educativo, que condicionan negativamente la presencia, el aprendizaje y participación de todo el alumnado, (Echeita et al., 2008).

Ainscow, Booth y Dyson (2006) definen la inclusión educativa como el proceso de avance sistemático que deben enfrentar las administraciones educativas y los centros escolares para tentar eliminar las barreras de distinto tipo que limitan la presencia, el aprendizaje y la participación del alumnado en la vida de los centros donde son escolarizados, con particular atención a aquellos más vulnerables. Presencia implica el lugar donde se escolarizan los alumnos/as y parece importante considerar que es difícil que las personas aprendan a reconocer el valor de 
la diversidad cuando se mantiene alejada de las clases ordinarias, en aulas y espacios segregados. Por aprendizaje debe entenderse que los alumnos/as con Trastorno del Espectro del Autismo tengan el mejor rendimiento escolar posible en todas las áreas del currículo de cada etapa educativa. Por participación debe reconocerse y apreciar la identidad de cada alumno/a y la preocupación en su bienestar personal, ausencia de situaciones de maltrato, exclusión y aislamiento social. Finalmente se entienden cómo facilitadores/barreras, entre otros/otras, los recursos, los servicios y las ayudas que prestan los centros para la inclusión, los valores de la cultura escolar, las políticas y las formas de concretar el currículo en el aula, todo lo cual puede llegar a condicionar positiva o negativamente la presencia, el aprendizaje o la participación de determinados alumnos en la vida escolar (Echeita et al., 2008).

La inclusión es ante todo un asunto de derechos humanos y por tanto asume la defensa de una educación en la que tienen cabida todas las personas, razón ética por la que debería también ser asumida por todos. Esta propuesta se sustenta en la premisa que en los centros educativos la educación debería satisfacer las necesidades de todos los individuos independientemente de sus particularidades y condiciones. Stainback y Stainback (1999) señalan que la inclusión es más que un método, una filosofía o un programa de investigación, siendo una forma de vivir. Tiene que ver con el vivir juntos/as; siendo una estrategia necesaria para analizar y comprender el proceso educativo para la vida en su complejidad (Echeita et al., 2008).

Los procesos inclusivos requieren el esfuerzo constante y no episódico, simultáneo y no descoordinado, de distintos agentes sociales: familias, profesionales, administración y sociedad en su conjunto (Verdugo \& Rodríguez, 2008). Sólo acciones bien coordinadas entre los profesionales, los centros educativos y la administración, junto a la participación familiar, garantiza el éxito y la permanencia de los cambios requeridos en los centros educativos (Verdugo \& Rodríguez, 2008).

Los cambios que se producen en la atención educativa al alumnado con Trastornos del Espectro del Autismo están relacionados con los cambios que se producen en la misma concepción de la alteración (Crespo, Campo, \& Verdugo, 2003; Luckasson et al., 1992, 2002; Organización Mundial de la Salud, 2001; Verdugo, 1994, 2003; Verdugo \& Rodríguez, 2008).
- El Paradigma de apoyos hace referencia a las posibilidades, las estimulaciones y la capacidad de poder aprender a vivir en comunidad y con los otros/as; si a esto añadimos la dificultad para aprenderlo, podemos entender la necesidad de aprenderlo en su contexto y ver su función de una manera clara y organizada. Los apoyos hacen mención al cubrimiento de estas necesidades y demandas que empiezan por la red natural de la persona (padres, familia, amigos) y sigue con los profesionales de los servicio/-s. Estas ayudas que necesitan las personas con Trastorno del Espectro del Autismo tienen el objetivo de las vidas que desean tener y que puedan sostener. El cambio se sintetiza en ver a estas personas no por sus dificultades sino por sus posibilidades (lo que pueden, pudieron y podrán hacer si se les brinda un entorno, una ayuda o el apoyo adecuado), más con lo que se puede hacer en función de los contextos que de los propios individuos exigiendo el apoyo en función de las necesidades y demandas; todos pueden hacer todo dependiendo del apoyo, su intensidad,....

Estos apoyos son definidos por Luckasson et al. (2002) como los recursos y estrategias destinados a mejorar el desarrollo, la educación, los intereses y el bienestar personal de una persona y que permiten su funcionamiento individual. Los apoyos son empleados para cumplir con diferentes funciones, pudiéndose emplear para aumentar las capacidades de una persona o para modificar los contextos para que potencien el funcionamiento del individuo. La idea de fondo que moviliza este énfasis en los apoyos es que cualquier persona con el apoyo adecuado y el tiempo suficiente mejorará, (Luckasson et al., 1992, 2002).

Los apoyos pueden servir también para disminuir la discrepancia entre las capacidades de las personas y las demandas de los entornos vivenciales. Estos entornos deben promover el bienestar de la persona, satisfacer sus necesidades y promover un sentimiento de estabilidad (Luckasson et al., 1992). Para ello, la evaluación y acopio de los servicios necesita centrarse en los apoyos que la persona desea y necesita en un ambiente más inclusivo, integrado y productivo (Luckasson et al., 2002) para lo que se ha de procurar dentro de la comunidad y que fomenten la participación activa en la misma, que promuevan la elección, que potencien el desarrollo de competencias y se basen en el respeto, (Luckasson et al., 1992). La evaluación del tipo e intensidad de las necesidades de apoyo de una persona 
son aspectos nucleares del sistema propuesto por la AAIDD y hacen referencia a los recursos de apoyo, las funciones de apoyo, la intensidad del apoyo y los resultados deseables (Schalock, 1999).

Desde mediados de los años ochenta, el paradigma de apoyos consigue, por lo menos, dos impactos significativos en las políticas y prácticas relativas a las personas con Trastorno del Espectro del Autismo. Primero, la orientación de apoyos agrupa (por lo general con el Plan Centrado en la Persona) las prácticas relacionadas de la planificación centrada en la persona, el crecimiento personal y oportunidades de desarrollar, la inclusión en la comunidad, la autodeterminación y la habilitación. Segundo, la implementación de apoyos individualizados lleva a esperar resultados personales mejorados, que generalmente se relacionan con las dimensiones básicas e indicadores de la calidad de vida (Schalock, 2009).

Los apoyos dentro del concepto de vida con apoyos se ofrecen, en un primer momento, dentro de la red natural de la persona: padres, familia, amigos, vecinos, colegas y voluntarios. Sólo cuando la red natural no puede ofrecer suficientes apoyos, entra en escena la red de seguridad social formada por los profesionales de los servicios. En muchos casos, resulta evidente que es necesario reconstruir o reactivar la red natural. Los apoyos deben ofrecerse de forma flexible: no todas las personas necesitan apoyos en las mismas áreas, en los mismos momentos o en igual cantidad, ofreciendo los apoyos que desean las personas para lo que es necesario interpretarlas, ayudarlas a expresarse y empoderarlas, (Van Loon, 2006)

Al proporcionar apoyos una organización debe concentrarse en maximizar la inclusión. Esto no implica una mayor dependencia en las redes sociales informales cómo familia, red de amigos, comunidad o voluntarios. Suponen optimizar los apoyos con el objetivo de eliminar los impedimentos para que las personas con Trastorno del Espectro del Autismo puedan desarrollar sus capacidades y asumir la gestión de sus vidas. La prestación de apoyos con el marco de calidad de vida desempeñan un claro avance de los resultados personales relacionados con la calidad de vida (Schalock, 2009; Van Loon, 2008).

- La Red de apoyo familiar y la búsqueda de la autodeterminación en las personas con trastorno del espectro autista: el futuro es hoy.
Cada vez es más evidente y nadie discute lo importante de incidir e involucrar a la familia en la educación de las personas, más si son diferentes (Mulas et al., 2010). El niño vive integrado en su ambiente familiar por lo que una adecuada información y educación redundará en un mayor aprendizaje con un menor grado de estrés e insatisfacción. La familia no sólo necesita un juicio clínico adecuado, precisa también información, educación para saber cómo interactuar con su hijo, apoyo por parte de las instituciones y solidaridad y comprensión por parte de la sociedad.

Por todo ello y en definitiva, lo que se pretende es ayudar a una persona con TEA a desarrollarse, a que alcance, él y su familia, la calidad de vida que todos merecemos y para ello es importante tener en cuenta, en palabras de Tamarit (1996) que para que las personas con TEA tengan conductas más flexibles, las personas que interactúen con ellos tienen que, también, mostrarles flexibilidad, es decir, darles posibilidad y diferencias para aprender de ellas y contrastar los comportamientos y actitudes de las diferentes personas y entornos que los forman. Generalmente, la interacción con personas con autismo sigue el siguiente esquema: el adulto comienza la interacción y el niño o la niña (o adolescente o adulto) responde; este esquema no responde a la realidad de los sistemas comunicativos e interactivos por lo que deben procurarse los modelos más eficaces para que las personas los entrenen y observen o viceversa.

Muy a menudo las acciones del adulto son órdenes para el niño (p.e., 'siéntate', 'vamos al baño', 'a comer', ...) por lo que deben considerarse su análisis y mejora. En este sentido, sería necesario crear entornos naturales y funcionales donde el esquema de interacción responda a realizar actividades y comunicaciones conjuntas donde prevalezca el todo con y no para o por la persona en desarrollo. En este sentido, la participación, la creencia en la persona, la sensación transmitida en su capacidad debe responder a empoderar a la persona para que se sienta capaz y partícipe de su aprendizaje y de las situaciones que lo median.

En el ámbito de los trastornos del espectro del autismo nos encontramos, con un gran número de investigaciones sobre la condición del Trastorno y un número mucho más escaso sobre las necesidades, demandas y atención de las personas que lo poseen. Nadie duda de la importancia del juicio clínico pero no podemos olvidarnos de la vida, el futuro, los recursos y 
las posibilidades de estas personas que tienen derechos y capacidades y no solo dificultades y alteraciones (Tamarit, 2005).

De hecho, en casi todos los documentos se observa a la patología más que la persona que necesita de los servicios, con sus necesidades, deseos e intereses más allá de lo que supone la alteración que presente; fruto de ello, se sigue percibiendo a la persona por sus carencias, por su curación y por sus dificultades obviando logros tan significativos dentro de la ciencia como que el TEA no es una enfermedad, caracteriza la forma de pensar pero no se puede curar, tiene un desarrollo característico y, al ser una minoría, son un colectivo en riesgo de exclusión. En definitiva una persona con sueños, con deseos, con esperanzas, con sentimientos y, sí, con limitaciones también, pero esencialmente con voz propia y con el derecho de todo ser humano a tener poder de decisión sobre su proyecto de vida (Tamarit, 2005).

Si algo caracteriza el comportamiento de estas personas, es la capacidad comunicativa disfuncional que poseen. Estando en una situación interpersonal, con otra persona o en referencia a otra persona, toda la conducta que tengamos es comunicativa. Cada comportamiento es una forma de comunicación. Cuando una persona muestra comportamientos que son difíciles o no deseables, comprender lo que está comunicando mediante el comportamiento es el paso más importante para poder lograr un cambio (Carr et. al, 1996). Moverse o quedarse quieto, hablar o permanecer callado, sonreír o mostrarse inmutable, presentarse o no a una cita, retirarse o quedarse en determinada situación, etc. constituyen comportamientos y tienen un efecto en la otra persona, por lo tanto, son comunicación. No sólo lo que se hace sino lo que se dice, los gestos, las posturas, el tono de voz también son comportamiento y lo que no se dice, no se gesticula o no se hace igualmente son comportamiento y en todos los casos tienen un valor comunicativo porque igualmente influye $o$ afecta a los otros. También la actividad comunicativa cumple con la función intrapersonal o de autorregulación cognitiva y conductual. En este sentido, desempeña un papel fundamental en el dominio emocional ya que facilita la capacidad para desarrollar el conocimiento de las emociones y su comprensión en una situación determinada (Baixauli, Roselló, \& Colomer, 2015).

La enorme heterogeneidad sintomática que presentan las personas con TEA también se refleja en el lenguaje y la comunicación, dando lugar a un espectro de funcionamiento que abarca desde la ausencia de conductas con intencionalidad comunicativa hasta el empleo de conductas de mayor complejidad funcional y formal (Martos \& Ayuda, 2002).

Tradicionalmente y hasta hace bien poco, una de las soluciones prioritarias se centraba en eliminar las conductas alteradas de dos maneras: explicándole al sujeto el porqué de la dificultad de su comportamiento o mediante el reforzamiento negativo. Lo que sucedía mayormente es que esta conducta se fortalecía y reforzaba más con su incremento y aprendizaje (Casey, López, \& Wacker, 2004). Desde hace unos años, aparece un nuevo paradigma del comportamiento centrado en la persona señalando que cada persona tiene sus particularidades a la hora de comportarse. Asimismo, el comportamiento está condicionado por la cultura donde vivimos. El hecho de calificar un comportamiento como asertivo, agresivo o inhibido no debe realizarse de forma taxativa. Hay culturas en las que se suele elevar más el tono de voz, o las personas se comunican situándose más cerca en el espacio. Asimismo, existen grandes variaciones culturales tanto en la clase como en su significado; también diferencias en función del rol que tengamos en un momento determinado; una persona se comunica de manera diferente si está con sus amigos, su familia, sus compañeros de trabajo, etc. Hablar de un comportamiento adaptado o no depende del contexto y de la persona con quien nos comunicamos. Esta perspectiva da lugar a una incipiente y cada vez más avanzada propuesta acerca de que estas conductas pueden ser un medio de comunicación en sujetos cuyas capacidades comunicativas presenten dificultades dado que el comportamiento es el instrumento más básico que los humanos utilizamos para comunicar. De ahí la importancia para proporcionar apoyo en la comunicación y favorecer su comprensión de la realidad que lo rodea; en ello, juega un papel muy importante la familia. Con una atención temprana la familia irá asimilándolo y facilitando su inclusión de una forma adecuada (Milla \& Mulas, 2009), de forma que se favorezca su desarrollo y aprendizaje.

La familia es para el sujeto su primer núcleo de convivencia y de actuación, donde irá modelando su construcción como persona a partir de las relaciones que allí establezca y, de forma particular, según sean atendidas sus necesidades básicas (Brazelton \& Greenspan, 2005). Este proceso de construcción de su identidad se 
dará dentro de un entramado de expectativas y deseos que corresponderán al estilo propio de cada núcleo familiar y social. Los padres como primeros educadores, en una situación "suficientemente" buena, establecerán un vínculo, una sintonía con el sujeto/a que les permitirá interpretar aquellas demandas de atención y de cuidado que precise su hijo/a en cada momento. Ellos serán los primeros responsables en la creación de unos canales y significación que favorecerán la construcción de la identidad del sujeto. López (1995) a partir de sus investigaciones sobre las necesidades de la infancia y la atención que éstas precisan afirma que:

"... Para la infancia no es adecuado cualquier tipo de sociedad, cualquier tipo de familia, cualquier tipo de relación, cualquier tipo de escuela, etc. sino aquéllas que le permiten encontrar respuestas a sus necesidades más básicas. El discurso de las necesidades es hoy especialmente necesario, porque no todos los cambios sociales que se están dando en la estructura familiar y en la relación padres e hijos están libres de riesgos para los menores" (p.9).

Las prácticas educativas parentales no sólo son la primera influencia para el niño/a, sino también la más significativa ya que muestran la manera en que los sujetos son educados y tratados por sus padres según algunas investigaciones como las de Ainsworth y Bell (1970); Schaffer y Crook (1981); Rodrigo y Triana (1985); Palacios y Oliva (1991); Goodnow (1996); Rodrigo y Palacios (1998); Barudy y Dantagnan (2005). Según Barudy y Dantagnan (2005) los buenos tratos, aseguran el buen desarrollo y el bienestar infantil y son la base del equilibrio mental de los futuros adultos y, por tanto, de toda la sociedad. El punto de partida de los buenos tratos a la infancia es la capacidad de madres y padres para responder correctamente a las necesidades infantiles de cuidado, protección, educación, respeto, empatía y apego. La competencia parental en estos aspectos vitales, permite que los niños/as puedan crecer como personas capaces de tener una buena autoestima y de tratar bien a los demás.

Por ello, la mayoría de los autores coinciden en la importancia de una intervención temprana que priorice el desarrollo de habilidades comunicativas espontáneas y funcionales así como hacer partícipe a los padres tanto a la hora de establecer objetivos, metas y prioridades, como de los resultados que se vayan obteniendo (Fortea, Escandell, Castro, \& Martos, 2015).

Los programas de atención deben atender esta situación y contexto de forma que disminuyan y afronten las barreras de estas personas en cuanto a presencia, aprendizaje y participación social y comunitaria además de mejorar sus posibilidades individuales de comprensión de la realidad social en la que viven, de comunicación y de aprendizaje (Milla \& Mulas, 2009).

Una buena intervención debe construir habilidades de comunicación espontánea y funcional, debe dar oportunidades para la comunicación a lo largo de todo el día y se puede llevar a cabo en diferentes contextos como puede ser el hogar, la escuela o cualquier otro contexto en el que el sujeto se sienta a gusto (Salvadó et al., 2012); todo ello redundará en crear una relación positiva donde su relación se caracterice por la cercanía, la empatía y el agrado mutuo (Carr et. al, 1996). El interés por actuar de forma espontánea y entusiasta con los demás determinará las relaciones interactivas de forma que se fomente y se entrenen. Para ello, al principio, se proporcionan situaciones sin condiciones (de forma no contingente) aprendiendo a asociar la interacción con experiencias positivas, interesantes y valiosas y a reconocer a la otra persona como alguien a quien merece la pena prestar atención; se reconocerá el éxito de esta actividad cuando la persona con Trastorno del Espectro del Autismo nos busque o muestre interés por la otra, es decir, parece que le agrada que esté cerca; las interacciones espontáneas, entusiastas y emocionalmente satisfactorias constituyen la base para el establecimiento de una relación positiva.

Posteriormente, es el momento de asegurarse que la persona inicie interacciones, que se acerque y comunique de la manera que le sea posible; de esta forma, estará aprendiendo que puede influir en los demás para conseguir cosas que le interesen sin recurrir a su comportamiento y llamadas de atención (Carr et al, 1996). Es el momento de enseñarle a esta persona a resultar agradable por lo que si pretendemos enseñar a comunicarse, tenemos que prestarnos como un oyente sensible $y$, para ello, necesitamos que la otra persona nos sea agradable. En nuestro mundo esto depende de la apariencia personal y los intereses compartidos por lo que tendremos que ocuparnos de estos aspectos; también, de establecer una relación empática con las personas que tutorizan, viven o se preocupan por su educación y desarrollo.

Cuando los problemas de comportamiento ocupan la función de comunicar es que resultan útiles al individuo y por ello lo manifiestan con 
frecuencia; al ocupar esta función comunicativa son difíciles de eliminar por lo que es preciso utilizar medios alternativos para proporcionar una alternativa para ello; cuando una conducta problemática y una conducta comunicativa tienen el mismo propósito y satisfacen el deseo o la intención de la persona podemos decir que son funcionalmente equivalentes por lo que requieren de una evaluación funcional; la información acerca del propósito nos permite elegir la conducta comunicativa que sustituya a la conducta problemática. Por ello no debemos contemplar los problemas de comportamiento como algo a eliminar tan rápidamente como podamos dado que son la fuente de información para procurar un medio comunicativo esencial (por ejemplo, puede que si le decimos a un sujeto que se golpea la cabeza para llamar nuestra atención que deje de hacerlo puede interpretar que no cumpla con la función de comunicar o que el propósito de su conducta no puede satisfacerlo). Si llegamos a comprenderlo, nos permitirá elegir la conducta funcionalmente equivalente que lo sustituya; para ello, es necesario enseñarlo mediante un entrenamiento general y extenso en comunicación (en vez de golpearte porque no me tocas en la espalda...); por ello, se puede decir que los intentos comunicativos no se trata de eliminarlos si no de que funcionen de forma adaptativa y nos permitan comunicarnos con los demás. Pero, para ello, es necesario acotar y determinar de una forma muy específica el propósito explícito que comunica y enseñarle a utilizarla de forma eficaz. Tener éxito en este proceso significa que la persona solicitará con más frecuencia, atención, ayuda y variedad de cosas concretas. Enseñar a comunicar significa reconocer las señales de nuestro entorno por lo que cuanto más comunique más señales conocerá y demandará. Esta eficacia en las formas de comunicación hacen referencia al propósito de comunicar permitiendo mayor facilidad en la realización y en la comprensión, es decir, debe ser fácil para la persona que lo realiza y debe permitir mayores niveles de comprensión en los demás ayudando a establecer interacciones eficaces y óptimas. Esto lleva consigo que la elección de las primeras formas de comunicación no sobrecarguen su capacidad de aprendizaje, es decir, que no requieran demasiado esfuerzo lo que le hará recurrir a medios ya conocidos y eficaces como la conducta problemática y, en segundo lugar, es necesario estimular el lenguaje comprensivo que supone comprender lo que los demás dicen y poder responder de una forma adecuada.

- Para elegir una forma de comunicación adecuada a la persona es necesario:

- Que la pueda utilizar en una amplia variedad de situaciones afines

- A ser posible que esté en el repertorio de la persona

- Que requiera poco esfuerzo debiéndola aprender de forma rápida con pocas ayudas y mostrarla adecuadamente.

- Para estimular su lenguaje expresivo, es necesario:

- Asegurarse de que la persona aprende una forma adecuada de comunicación expresiva que sustituya a la conducta problemática

- Comprobar la utilidad de la comunicación en su funcionamiento.

- Evaluar las habilidades de lenguaje comprensivo.

- Enseñar habilidades de comprensión relacionadas con los problemas de comportamiento

- Supervisar las interacciones comunicativas de mayor duración así como las situaciones concretas en que se utilizan.

La recogida rigurosa de datos y su análisis proporciona el rigor para proceder a establecer sistemas de comunicación funcionales y eficaces. Los estudios de campo no permiten realizarlos de forma tan rigurosa como la literatura experimental exige dado que se procede a mejorar la vida de las personas y sus habilidades para convivir en sus entornos más o menos próximos pero cotidianos; ello exige la puesta en marcha de planteamientos más asequibles que los experimentales. Lo que se busca en este planteamiento es hacerlo con el mayor rigor posible pero persiguiendo el éxito de los programas de intervención. La base científica de la que partimos y en la que se basan estos procesos es fuerte como hemos descrito previamente por lo que la utilización de un procedimiento que aumente las exigencias del profesional y revierta en menor atención y garantías de éxito en las personas no significa ser menos rigurosa si no en vigilar que este proceso se realice de forma que redunde en beneficio de las personas para las que es construido y en función de las que se realiza. Este enfoque asequible persigue la satisfacción del demandante o consumidor que se describe en terminología científica como validez social; una validez social pobre (baja satisfacción del consumidor) significa que algo no funciona correctamente por lo que es preciso una eva- 
luación de mayor profundidad. Por otro lado, la satisfacción del consumidor no es garantía del buen trabajo y esfuerzo en la consecución de los objetivos planteados en función de la demanda dado que muestra un juicio subjetivo que implica muchas consideraciones como empatía, percepción, paciencia, comunicación... pero ello no significa ningún desmerecimiento de la misma, simplemente que debe analizarse de forma que redunde en la mejora de los procedimientos y recursos para la atención para una vida independiente y autónoma de las personas con TEA con respecto al mundo diferente en el que viven y deben convivir con y entre los demás. Por cierto, este mundo futuro nadie sabe como será y lo hacemos proyectando nuestras perspectivas acerca del mismo y de la ilusión de vida de estas personas en él; muchas veces, esa ilusión nos hace perder la perspectiva del ahora; el ser humano es un ser social necesita de los demás y tiene que aprender a vivir con las demás personas en un mundo donde todos y todas tengamos cabida; ese es el reto y esa es nuestra posibilidad de vida como especie independientemente y teniendo en cuenta toda nuestra diversidad que nos enriquece y hace mejores.

\section{Referencias}

Aierbe, A. (2005). Intervención psicopedagógica en los trastornos del desarrollo. Málaga: Ediciones Aljibe.

Ainscow, M., Both, T., \& Dyson, A. (2006). Improving schools, developing inclusion. London: Routledge

Ainsworth, M.D. \& Bell, S.M. (1970). Apego, exploración y separación, ilustrados a través de la conducta de niños de un año en una situación extraña. En: J. Delval (comp.) Lecturas de psicología del niño, Vol. 1,1978. Madrid: Alianza.

Asociación Americana de Psiquiatria (2014). Guía de consulta de los criterios diagnósticos del DSM5. Barcelona: Masson.

Alba, C. (1994). Utilización didáctica de recursos tecnológicos como respuesta a la diversidad. En J.M Sancho (coord.), Para una tecnología educativa. Barcelona: Hosori.

Baixauli, I., Roselló, B.\&y Colomer, C. (2015). Relaciones entre trastornos del lenguaje y competencia socioemocional. Revista de Neurología, 60, S61-S56.

Barudy, J.\& Dantagnan, M. (2005). Los buenos tratos a la infancia. Barcelona: Gedisa.

Brazelton T. \& Greenspan S. (2005). Las necesidades básicas de la infancia. Barcelona: Graó.

Bronfenbrenner, U. (1979). The ecology of Human Development. Cambridge. Harvard University Press. (Trad. Cast.: La ecología del desarrollo humano. Barcelona, Ediciones Paidós, 1987).

Carr, E. G., Levin, L., McConnachie, G., Carlson, J. I., Kemp, D.C. \& Christopher E. (1996). Intervención comunicativa sobre los problemas de comportamiento. Madrid: Alianza.

Casey, S. D., López, J. C., \& Wacker, D. P. (2004). Evaluación funcional del comportamiento en personas con discapacidades del desarrollo. Revista Latinoamericana de Psicología, 36(2), 269-287.
Crespo, M., Campo, M., \& Verdugo, M.A. (2003). Historia de la clasificación internacional del funcionamiento de la discapacidad y de la salud (CIF). Un largo camino recorrido. Siglo Cero, 34(1), 2026.

Cunningham, C., \& Davis, H. (1988). Trabajar con los padres. Marcos de colaboración. Madrid: Siglo XXI y Ministerio de Educación y Ciencia.

Echeita, G. (2008). Inclusión y exclusión educativa. "Voz y quebranto". REICE - Revista Electrónica Iberoamericana sobre Calidad, Eficacia y Cambio en Educación 2008, 6(2), 9- 18.

Echeita, G. et al. (2008). La inclusión educativa del alumnado con necesidades educativas especiales, asociadas a discapacidad, en España. Un estudio prospectivo y retrospectivo de la cuestión, vista desde la perspectiva de las organizaciones no gubernamentales de personas con discapacidad, Informe de investigación no publicado.

Fortea, M.S., Escandell, M.O., Castro, J.J., \& Martos, J. (2015). Desarrollo temprano del lenguaje en sujetos pequeños con trastorno del espectro autista mediante el uso de sistemas alternativos. Revista de Neurología, 60, S31-S35.

Freixa, M. (1993). Familia y Deficiencia mental. Salamanca: Amarú.

Frith, U. (1991). Asperger and his syndrome. En U. Frith (Ed.), Autism and Asperger Syndrome. (pp. 1-36). Cambridge: Cambridge University Press.

Frith, U. (2003). Development and neurophysiology of mentalizing. Philosophical Transactions of the Royal Society of London, 358, 459-473.

Gallimore, R., Coots, J.,Weisner, T.S., Garnier, H., \& Guthrie, D. (1996). Family responses to children with early developmental delays II: Accomodation intensity and activity in early and middle childhood. American Journal on Mental Retardation, 101(3), 215-232.

Gallimore, R., Weisner, T.S., Bernheimer, L.P., Guthrie, D., \& Nihira, K. (1993). Family responses to young children with developmental delays: Accomodation activity in ecological and cultural context. American Journal on Mental Retardation, 98(2), 185-206.

Gallimore, R., Weisner, T.S., Kaufman, S.Z., \& Bernheimer, L.P (1989). The social construccion of ecocultural niches: Family accomodation of developmentally delayed children. American Journal on Mental Retardation, 94(3), 216-230.

García Sánchez, J. (2005). Intervención psicopedagógica en los trastornos del desarrollo. Madrid: Pirámide.

Giné, C. (2001). Familia e intervención precoz. Orientaciones para la intervención del psicopedagogo. En B. Del Rincón Igea, Presente y futuro del trabajo psicopedagógico. Barcelona: Ariel

Goodnow, J.J. (1996). From household practices to parents'ideas about work and interpersonal relationships. Harkness \& Super (eds.). Parents'cultural belief systems. Their origins, expressions and consequences. New York: Guildford.

Guralnick, M.J. (Ed.) (1997). The effectiveness of Early Intervention. Baltimore: Paul H. Brookes, Pub.Co.

Guralnick, M. J. (1998). The effectiveness of early intervention for vulnerable children: A developmental perspective. American Journal on Mental Retardation, 102, 319-345.

Heward, W.L. (2000). Niños excepcionales. Una introducción a la educación especial. Madrid: Prentice Hall.

Kebbon, L. (1993). Pupils With Mild Mental Retardation in Regular Swedish Schools: Prevalence, Objective, Characteristics, and Subjective. Evaluations. Swedish Children With Mild Mental Retardation, 976),692-701.

Lambert, J. \& Rondal, J.A. (1982). El mongolismo. Barcelona: Herder 
López, F. (1995). Necesidades de la infancia: Respuesta familiar. Infancia y Sociedad, 30, 8-47.

Luckasson, R., Borthwick-Duffy, S., Buntinx, W.H.E., Coulter, D.L., Craig, E.M., Reeve, A.,... Tassé, M.J. (2002). Mental retardation: Definition, classification, and systems of supports (10a ed.).Washington (DC): American Association on Mental Retardation. [Traducción castelllana de M.A. Verdugo y C. Jenaro. (2003) Madrid: Allianza Editorial].

Luckasson, R., Coulter, D. L., Polloway, E. A., Reiss, S., Schalock, R. L., Snell,... Stark, J. A. (1992). Mental retardation: Definition, classification, and systems of supports (9a ed.). Washington, DC: American Association on Mental Retardation.

Martos, J. \& Ayuda, R. (2002). Comunicación y lenguaje en el espectro autista: el autismo y la disfasia. Revista de neurología, 34, S58-S63.

Mesibov, G. (1990). Normalisation and its relevance today. Journal of Autism and Developmental Disorder, 20, 379-390

Millá, M.G. \& Mulas, F. (2009). Atención temprana y programas de intervención específica en el trastorno del espectro autista. Revista de Neurología, 48, S47-S52.

Molina, S. (1999). Deficiencia mental. Aspectos evolutivos y educativos. Málaga: Aljibe

Mulas, F.; Ros-Cervera, G.; Millá, M.; Etchepareborda, M.; Abad, L., \& Téllez, M. (2010). Modelos de intervención en niños con autismo. Revista de Neurología, 50 (3), 577-584.

Organización Mundial de la Salud (2001). Informe sobre la salud en el mundo 2001 - Salud mental: nuevos conocimientos, nuevas esperanzas. Francia. Recuperado de http://www.who.int/whr/2001/en/ whr01 es.pdf?ua=1

Palacios, J., \& Oliva, A. (1991). Ideas de madres y educadores sobre la educación infantil. Madrid: MEC.

Paniagua, G. (1999). Familias con niños con necesidades educativas especiales. En A. Marchesi, C. Coll e J. Palacios, Desarrolo psicológico y educación 3. Trastornos del desarrollo y necesidades educativas especiales (pp. 469-493). Madrid: Alianza.

Perrin, B., \& Nirje, B. (1985). Setting the record straight: a critique of some frequent misconceptions of the normalization principle. Australia and New Zealand Journal of Developmental Disabilities, 11(2), 69-74.

Riviere, A. (2000). ¿Cómo aparece el atismo? Diagnóstico temprano e indicadores precoces del trastorno autista. En A. Riviere y J. Martos (comp.), El niño pequeño con autismo. Madrid: APNA.

Rodrigo, M.J. \& Palacios, J. (coords.) (1998). Familia y desarrollo humano. Madrid: Alianza Editorial.
Rodrigo, M.J. \& Triana, B. (1985). El concepto de infancia en nuestra sociedad: una investigación sobre teoría implícita de los padres. Infancia y Aprendizaje, 3132, 165-172.

Salvadó, B., Palau Baduell, M., Clofent Torrentó, M., Montero Camacho, M., \& Hernández Latorre, M.A. (2012). Modelos de intervención global en personas con trastorno del espectro autista. Revista de Neurología, 54, 63-71.

Schaffer, H.R., \& Crook, Ch.K. (1981). El papel de la madre en el desarrollo social temprano. Infancia y aprendizaje, 15, 19-37.

Schalock, R. L. (1999). Hacia una nueva concepción de la discapacidad. Siglo Cero, 30(1), 5-20.

Schalock, R. (2009). La nueva definición de discapacidad intelectual, apoyos individualesy resultados personales. Siglo Cero, 40(1), 22-39.

Stainback, S. \& Stainback, W. (1999). Aulas Inclusivas. Madrid: Narcea, S. A. de Ediciones.

Tamarit, J. (1996) Acerca de la insistencia en la igualdad: Análisis y propuestas para la intervención. 5th Congress AUTISME-EUROPE. Barcelona.

Tamarit, J. (2005) Autismo: modelos educativos para una vida de calidad. Revista de Neurología, 40. 181-186.

Taylor, S. \& Brown, J. (1988). Illusion and Well-Being: A Social Psychological Perspective on Mental Health. Psychological Bulletin, 103(2), 193-210.

Tomkiewicz, S. (1979). Relación del débil mental con su familia. Siglo Cero, 66, 12-29.

Van Loon, J. (2008). Aligning quality of life domains and indicators to support intensity scale companion guide: A resource for SIS users (pp. 80-87). Washington, DC:

American Association on Intellectual and Developmental Disabilities.

Verdugo, M.A. (1994). El cambio de paradigma en la concepción del retraso mental: la nueva definición de la AAMR. Siglo Cero, 25(5), 5-24.

Verdugo, M.A. (2003). Análisis de la definición de discapacidad intelectual de la Asociación Americana sobre retraso mental de 2002. Siglo Cero, 34(1), 5-19.

Verdugo, M. A., \& Rodríguez Aguilella, A. (2008). Valoración de la inclusión educativa desde diferentes perspectivas. Siglo Cero, 39(4), 5-25.

West, M. A. (1986). El impacto del retardo mental sobre la familia: implicaciones para la prevención. Siglo Cero, $108,50-56$

Wolfensberger, W. (1972). The principle of Normalization in human services. Toronto:National Institute on Mental Retardation.

Para citar esta comunicación:

Baña Castro, M. (2015). El rol de la familia en la calidad de vida y la autodeterminación de las personas con trastorno del espectro del autismo. Ciencias Psicológicas, 9(2), 323 - 336. 